Techniques \& Culture

\title{
Dire le droit local de l'eau
}

Narrating water rules and rights

Jeanne Riaux

\section{(2) OpenEdition}

Journals

Édition électronique

URL : https://journals.openedition.org/tc/5659

DOI : $10.4000 /$ tc. 5659

ISBN : 1952-420X

ISSN : 1952-420X

Éditeur

Éditions de l'EHESS

Édition imprimée

Date de publication : 15 décembre 2011

Pagination : 166-181

ISBN : 978-2-7351-1437-5

ISSN : 0248-6016

\section{Référence électronique}

Jeanne Riaux, « Dire le droit local de l'eau », Techniques \& Culture [En ligne], 57 | 2011, mis en ligne le 30 juin 2012, consulté le 29 septembre 2022. URL : http://journals.openedition.org/tc/5659 ; DOI : https:// doi.org/10.4000/tc.5659 


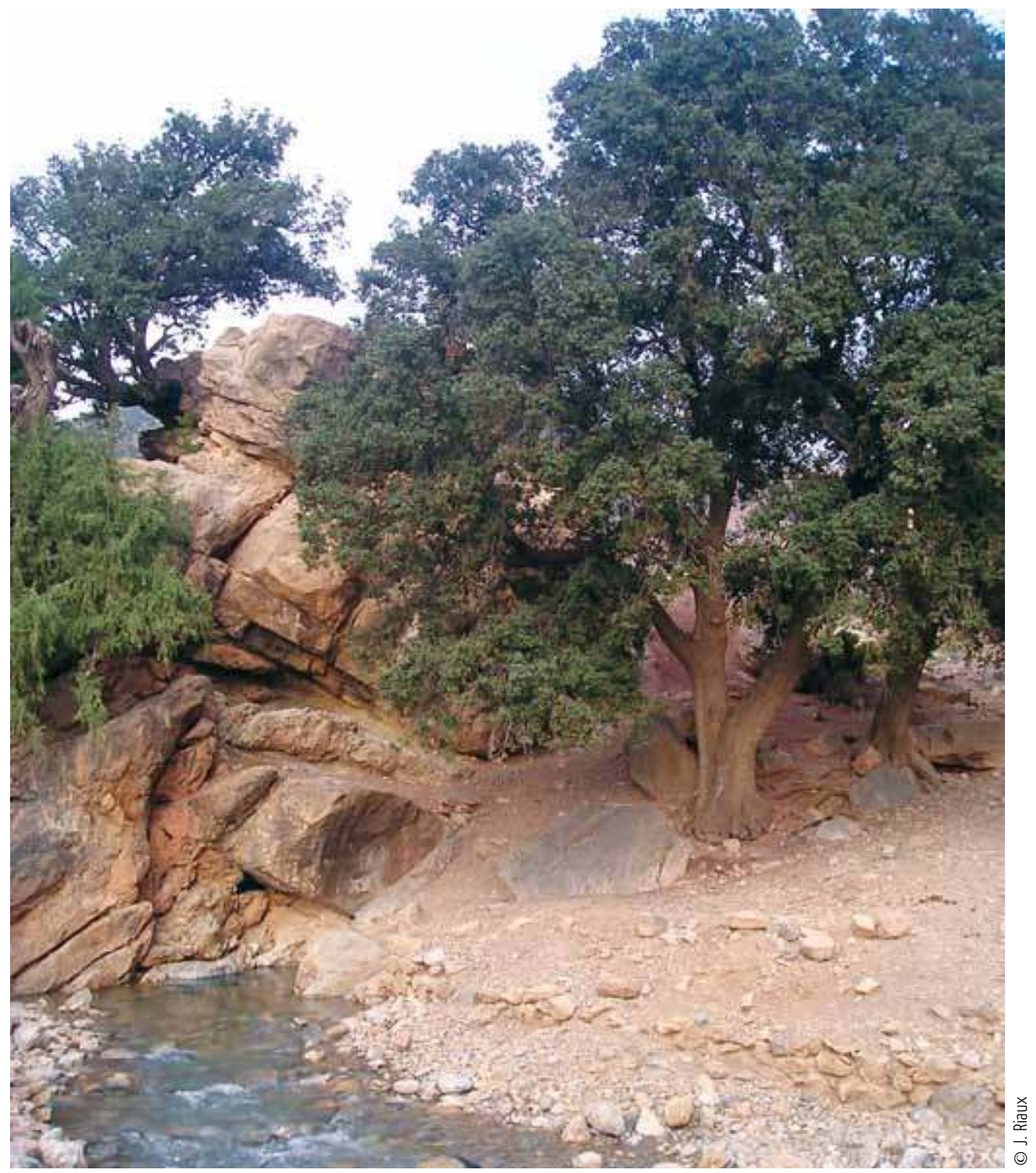




\section{Jeanne Riaux}

IRD-UMR G-EAU

jeanne.riaux@ird.fr

Techniques \& Culture 57, 2011/2: 166 - 181

\section{DIRE LE DROIT LOCAL DE l'EAU}

\section{Histoire orale et partage de l'eau dans la vallée des Aït Bou Guemez (Haut Atlas, Maroc)}

La gestion de l'eau dans les sociétés paysannes suscite de nombreuses interrogations dont un bon nombre porte sur l'appropriation et le partage des eaux. La variété des droits, règles et normes relatifs à l'eau et celle de leurs agencements a été amplement décrite et analysée.

Les recherches sont d'abord centrées sur des communautés relativement autonomes par rapport aux pouvoirs centraux. Il s'agit de valoriser le droit des communautés dont l'importance est souvent occultée par les juristes et par les États (Tozy \& Mahdi 1990). Le droit mis en œuvre localement ne peut être réduit au droit coutumier tel qu'il est formalisé en marge de certains corpus de lois. Assier-Andrieu (1980) souligne ce constat en introduisant la notion de droit local, comme pratique sociale des communautés exprimée notamment dans le domaine de la gestion des ressources collectives. L'étude du droit local met l'accent sur le pluralisme légal et sur la manière dont les corpus de droits et de normes d'origines diverses s'articulent au cours du temps et s'ajustent à des contextes spécifiques (Bédoucha 2000). Ces recherches conduisent à l'examen des relations existant entre le droit définit comme un ensemble de règles normatives et les pratiques effectives (Ostrom 1992). Certains auteurs analysent les pratiques des individus et des groupes autour de différents registres de droit (Lecestre-Rollier 1999) ou de justification et de légitimité (Ruf 1995) ; le droit local apparaît produit et mobilisé en fonction d'événements et de rapports sociopolitiques. Les interactions entre États et communautés, notamment dans le cadre de la mise en conformité des pratiques locales avec le droit national, font ainsi l'objet d'une attention particulière (Bruns \& Meinzen-Dick 2000, Boelens \& al. 2007). 
Cette dernière thématique fait écho aux préoccupations contemporaines des communautés d'irrigants et des agents de développement. Depuis une vingtaine d'années, les questions de création d'associations d'usagers et d'optimisation du partage de l'eau sont à l'honneur avec la mise en œuvre de politiques dites participatives. L'analyse des pratiques locales et des corpus de droits qui les sous-tendent doit donc être poursuivie afin d'accompagner les processus de changement en cours. C'est le sens des recherches entreprises dans la vallée des Ait Bou Guemez (Figure 1) alors que la formalisation des droits et la modernisation des réseaux hydrauliques par l'État étaient à l'œuvre depuis 1999. Les agents en charge du projet proposaient de formaliser les règles existantes sans les modifier. Malgré cette volonté de maintien du droit local, on observe des contradictions entre règles formalisées et règles mises en œuvre localement (Riaux 2006).
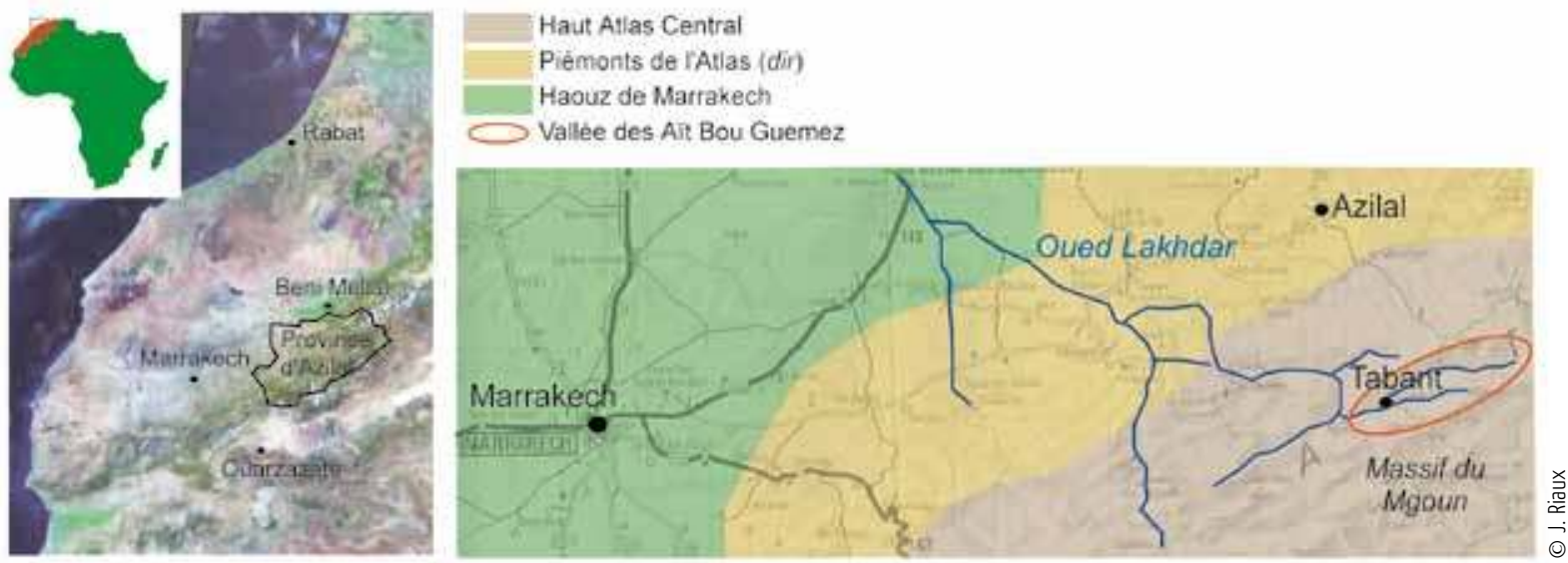

\section{Localisation de la vallée \\ des Aït Bou Guemez}

Haut Atlas central, Maroc.

(Fig. 1)
Ces contradictions résultent pour partie d'une focalisation excessive sur les prescriptions opérationnelles ou règles mises en pratique. Les règles qui encadrent ces pratiques sont difficilement observables et ne font pas l'objet d'énoncés prescriptifs spécifiques ; elles n'ont pas été prises en compte dans les règlements écrits (Riaux 2011). Pourtant ces règles sont au centre d'un ensemble de récits que les Ait Bou Guemez mettent en avant lorsqu'ils racontent leur histoire.

Dans une société où le droit de l'eau demeure majoritairement oral, les récits constituent un mode d'énonciation du droit qui doit être considéré en tant que tel. Nous proposons de restituer et d'étudier ces récits, démarche classique en anthropologie mais peu mobilisée dans le domaine de l'eau. Il s'agira d'interroger les modalités d'énonciation et de justification d'un droit formel tel que le présentent les Ait Bou Guemez à travers l'histoire du partage de l'eau. 


\section{Des groupes sociohydrauliques}

Les Ait Bou Guemez, sont installés dans des villages situés en bordure de périmètre irrigué (Figure 2). Les territoires villageois sont disposés de manière transversale à l'axe de la vallée, si bien que leurs habitants ont accès à l'ensemble des ressources agro-écologiques étagées du fond de vallée jusqu'aux sommets (Figure 3). L'accès à ces ressources diversifiées permet une complémentarité des activités productives sur laquelle repose la gestion des aléas climatiques et politiques (Garrigues-Cresswell $\&$ Lecestre-Rollier 2001). Mais on observe à travers l'histoire récente un repli des activités sur le fond de vallée et sur l'usage des ressources en eau. En plus de ses fonctions domestiques et vivrières, l'eau a désormais un rôle économique accru avec l'essor de l'élevage et de l'arboriculture intensifs.

L'importance de l'eau, de son partage et de ses usages, est ancrée dans l'espace. L'ensemble du fond de vallée est dessiné par la pratique de l'irrigation (Figure 4), celle-ci étant effectuée à partir d'une trentaine de résurgences karstiques de régime pluvio-nival qui s'écoulent dans un assif (oued) avant d'être détournées vers les canaux d'irrigation. Le territoire est ainsi alimenté par une multitude de sources dont le débit fluctue au fil des saisons. La relation aux sources est l'un des éléments structurant le découpage territorial du fond de vallée, ce que la notion d'aire sociohydraulique met en exergue. L'aire sociohydraulique est un espace approprié, aménagé et irrigué à partir d'une unique source par plusieurs groupes villageois ;

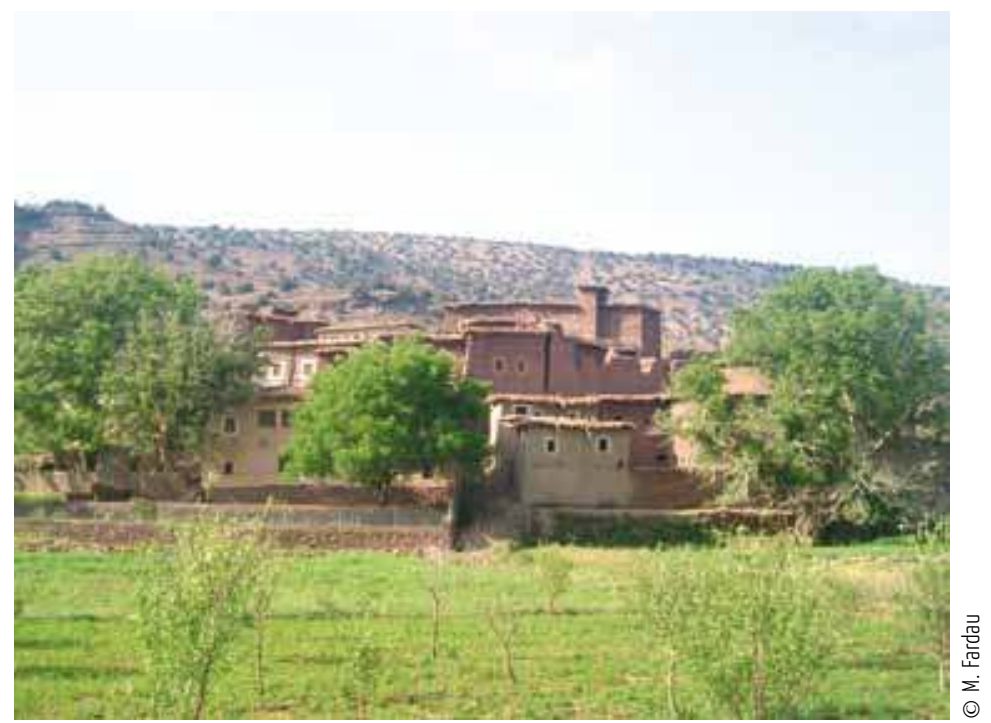

Village d'Ibaqalliwn, situé en bordure de l'espace irrigué. (Fig. 2)

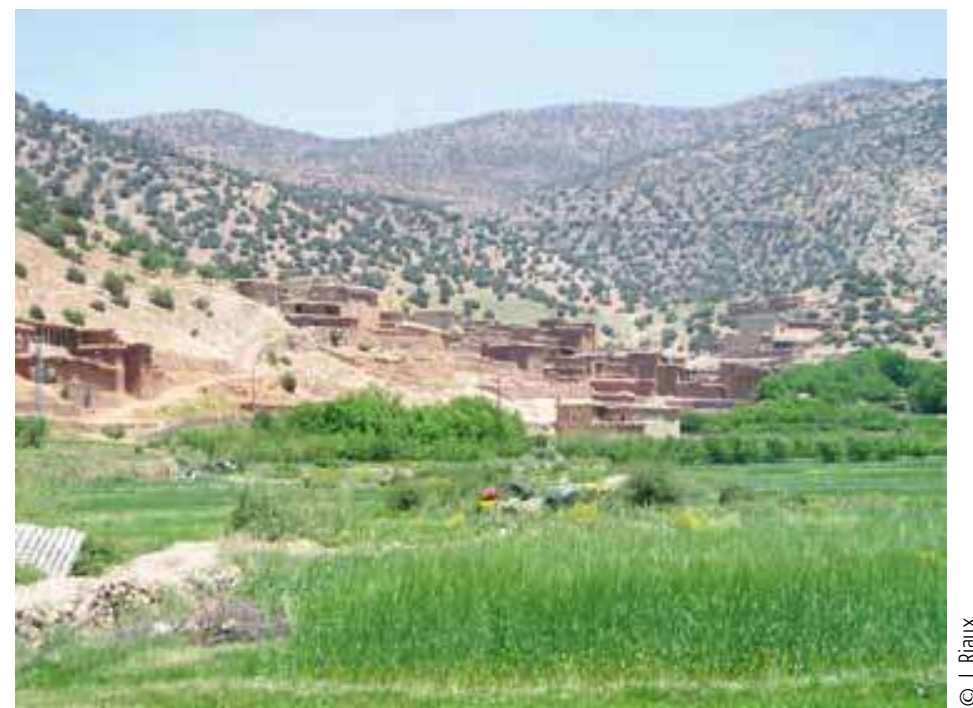

Le village de Rbat et l'étagement des ressources agro-écologiques

Dans le fond de vallée, les terres arrosées permettent de réaliser deux à trois récoltes par an. Au-dessus des canaux, la zone de cultures pluviales est semée de lentilles ou de céréales. En remontant les versants, les zones villageoises de forêts sont pâturées et fournissent le bois de chauffe. Les pâturages collectifs sont situés plus haut. (Fig. 3) 


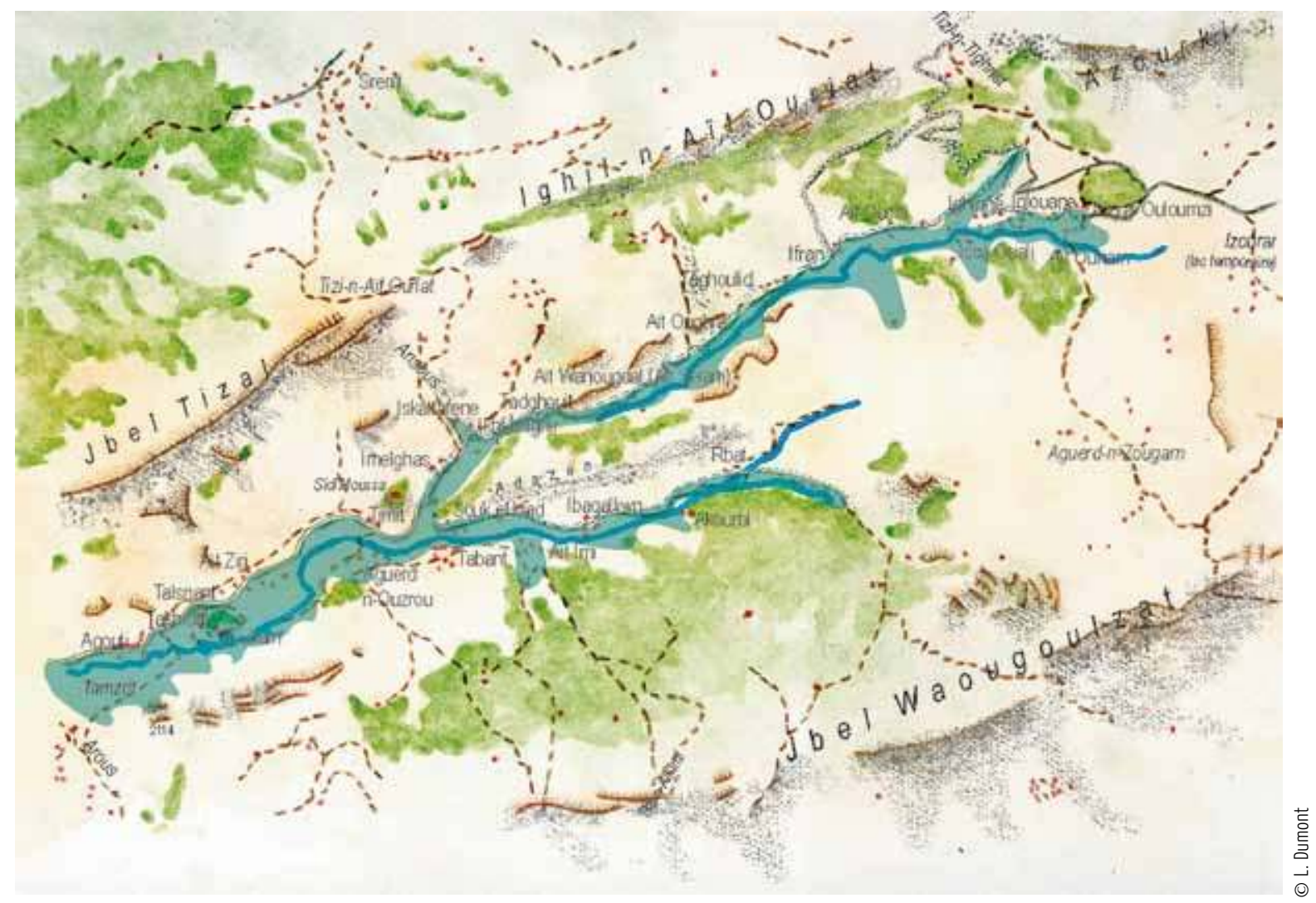

\section{La vallée des Aït Bou Guemez, vaste espace irrigué dans le Haut Atlas}

Chacune des sources qui irriguent la vallée est appropriée par

plusieurs groupes villageois. Les groupes sociohydrauliques, ainsi formés par et pour l'usage des eaux d'une source, relatent l'histoire de l'appropriation et du partage des eaux à travers des récits. Le travail proposé concerne les eaux de la source de Rbat, en amont du vallon de Rbat-Tabant [Dessin d'après IGN].

(Fig. 4)

le groupe ainsi formé par et pour l'usage de l'eau est un groupe sociohydraulique (Riaux 2006). Les aires sociohydrauliques sont le fruit de la rencontre entre les caractéristiques physiques d'un espace et son organisation sociotechnique, notamment avec les règles de définition du groupe d'ayants droit aux eaux de chaque source et avec la construction de canaux permettant de diriger l'eau sur l'espace défini comme ayant droit.

Chaque groupe sociohydraulique a une histoire commune et c'est à travers elle que les Ait Bou Guemez racontent l'origine du partage de l'eau. L'histoire du groupe sociohydraulique de la source de Rbat est relatée grâce à deux principaux récits ${ }^{1}$ : la fondation mythique des villages de l'aire sociohydraulique ${ }^{2}$ et la pacification de la vallée durant le Protectorat. 


\section{Aux origines du groupe sociohydraulique}

Les anciens racontent qu'à l'origine le vallon de Rbat jusqu'à Ibaqalliwn était une forêt dense peuplée d'animaux sauvages (Figure 5). À l'époque, des éleveurs du sud menaient chaque été leurs troupeaux dans les pâturages du Haut Atlas, dont la vallée des Ait Bou Guemez. Des nomades Ait Atta décidèrent de laisser chaque année quelques hommes

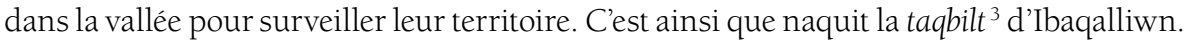
Ils s'installèrent près d'une source avec quelques bêtes et cultivèrent les terres. Plus tard, ils firent venir des femmes, se marièrent et s'installèrent définitivement. Ibaqalliwn crût progressivement au rythme des mariages et des naissances. Selon les anciens, cinq lignages ${ }^{4}$ descendent de ces premiers occupants.

Un jour, un jeune berger arrivé d'ailleurs proposa ses services à la taqbilt d'Ibaqalliwn qui accepta. Chaque matin il partait avec le troupeau des Ait Ibaqalliwn et le menait pâturer dans la forêt en amont du village. Un soir, au moment de rentrer, le berger s'aperçut que l'une des chèvres avait la barbichette perlée de gouttes d'eau. Le lendemain, il la suivit et vit la chèvre creuser le sol et l'eau jaillir (voir photo d'ouverture). Le berger garda sa découverte secrète. Quelques temps après, les Ait Ibaqalliwn lui demandèrent ce qu'il souhaitait en paiement de ses années de travail. Le berger demanda le territoire de forêt situé en amont du village. Son souhait fut accepté. Il s'installa sur ce territoire, se maria et eut des enfants. Il fit aussi venir des personnes de l'endroit d'où il venait. «C'étaient des bergers, mais quand ils ont découvert la source, ils sont devenus des agriculteurs » explique-t-on. Les nouveaux arrivants s'organisèrent en deux groupes occupant chacun une moitié du village. C'est ainsi que fut créée la taqbilt des Ait Rbat, divisée en deux groupes distincts jusqu'à l'heure actuelle.

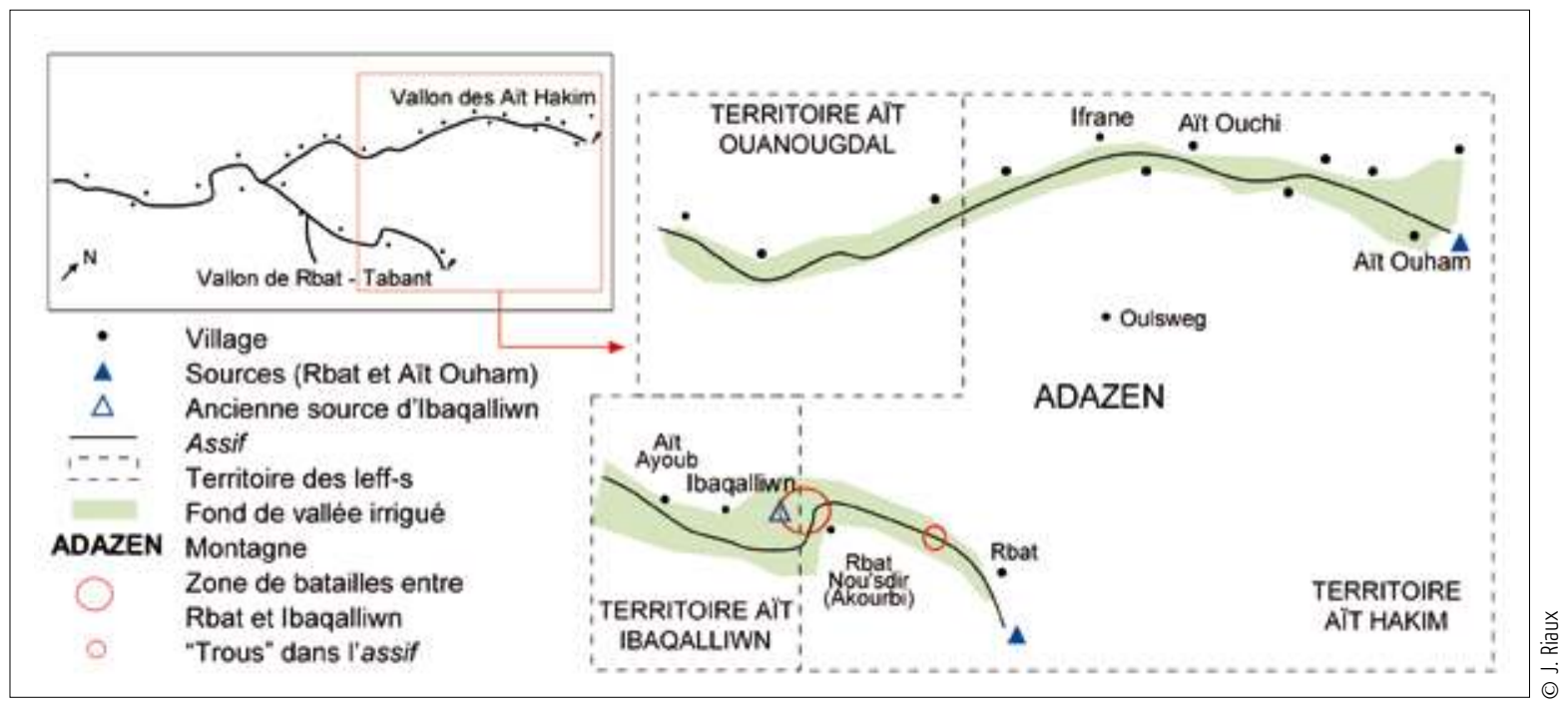

«à l'origine était une forêt peuplée d’animaux sauvages ... » : les lieux du récit

l'histoire du partage des eaux de la source de Rbat fait référence à un espace physique et social plus large que le seul territoire des ayants droit de la source de Rbat. (Fig. 5) 
Le tour d'eau intervillageois

D'après les récits, le partage de l'eau a été ajusté avec l'évolution des découpages sociopolitiques du territoire. Ainsi présenté, ce partage apparaît très égalitaire.

(Fig. 6)
Plus tard, les Ait Ibaqalliwn se rendirent compte qu'ils avaient donné au berger un territoire comprenant une source. En effet, les premiers Ait Rbat avaient entrepris de couper les arbres de la forêt et de cultiver leur terroir. Ils l'arrosaient avec les eaux de la source. Selon les anciens, le territoire de Rbat s'est peu à peu étendu jusqu'à atteindre les parcelles d'Ibaqalliwn. C'est alors que la source d'Ibaqalliwn tarit. Les Ait Ibaqalliwn voyant les parcelles verdoyantes de leurs voisins ne tardèrent pas à découvrir ce que le berger leur avait caché : ils avaient donné avec le territoire d'amont une source généreuse. Dès lors, ils accusèrent le berger de traîtrise et entrèrent en guerre avec les Ait Rbat. D'autant que ces derniers, de plus en plus nombreux, commençaient à menacer le territoire des Ibaqalliwn. Pour parer aux attaques des Aitt Ibaqalliwn, les Ait Rbat fondèrent un hameau défensif à la frontière des territoires de Rbat et Ibaqalliwn. Pour peupler ce hameau, ils y installèrent des familles travaillant à leur service. Elles purent peu à peu acheter et cultiver des parcelles sur l'aval du territoire de Rbat. Ainsi fut fondé Rbat nou'sdir (Rbat d'en bas). Par la suite, ce hameau devint Akourbi. Mais à cette époque là, Rbat d'en bas fut le théâtre d'incessants affrontements entre les Ait Rbat et les Ait Ibaqalliwn. Selon les anciens, la dernière guerre entre Rbat et Ibaqalliwn dura sept ans. Les Ait Rbat creusèrent un trou dans le lit de l'assif où toute l'eau de la source s'engouffrait avant d'arriver à Ibaqalliwn. Privés d'eau, les Ait Ibaqalliwn vécurent sept ans de sécheresse. Les troupeaux périrent, les champs étaient secs, aucune récolte ne fut faite.

À cette époque, Ibaqalliwn, Ifrane et les Ait Ouanougdal, situés dans le vallon des Ait Hakim, formaient un groupe d'alliés (leff). Aussi, après de multiples tentatives guerrières, les Ait Ibaqalliwn eurent l'idée de demander secours à leurs alliés. Ils partirent avec un bœuf à sacrifier à la taqbilt d'Ifrane. Comme ils gravissaient la montagne Adazen, ils virent les parcelles verdoyantes de Rbat. Un ancien demanda alors « pourquoi aller chercher de l'aide à Ifrane? Au mieux ils nous donneront un peu de céréales et le problème ne sera pas résolu ». Après concertation, les Aitt Ibaqalliwn rebroussèrent chemin. Une délégation de la taqbilt se rendit à Rbat où fut sacrifié le bœuf. Par cet acte, les Ait Ibaqalliwn demandaient la paix aux Ait Rbat qui acceptèrent. Les eaux de la source furent partagées entre les deux taqbilts. Selon certains, il y aurait eu un acte écrit pour sceller cet accord en vertu duquel

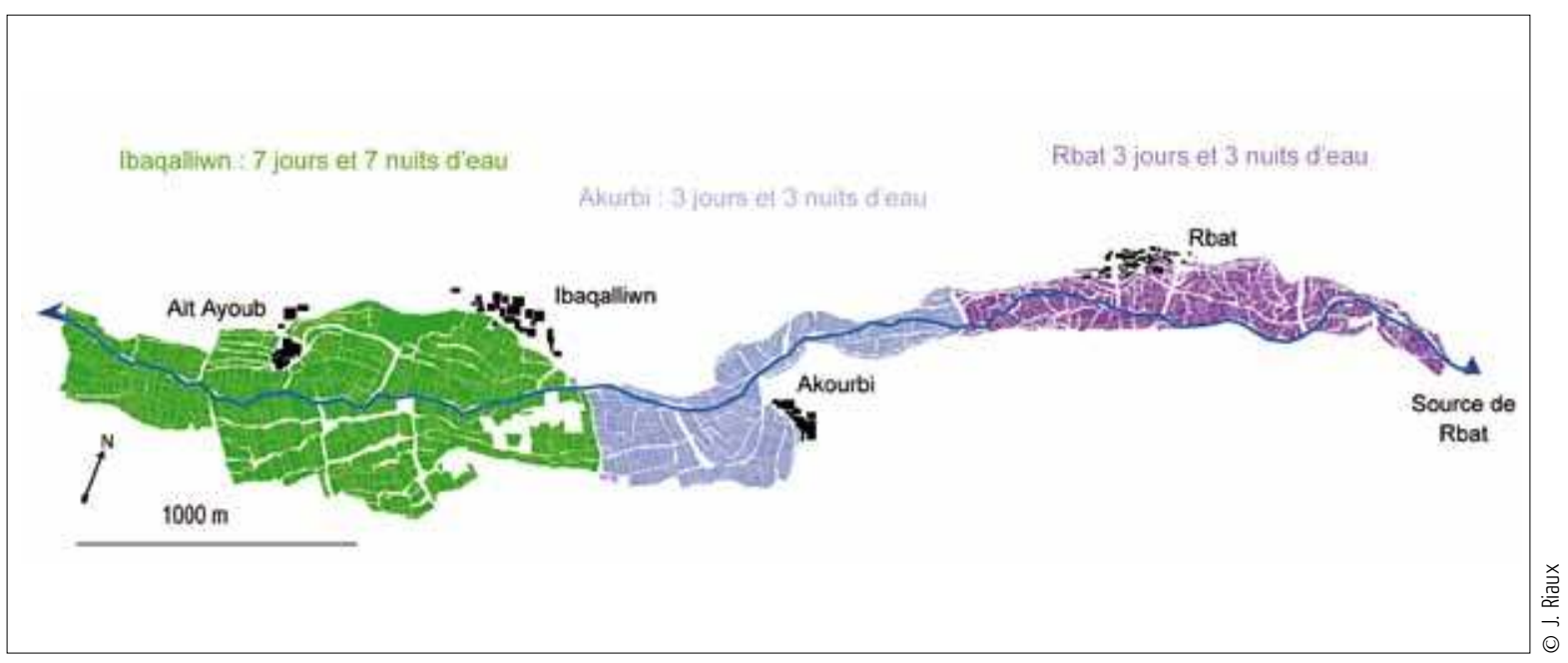


les habitants de Rbat eurent le droit d'utiliser l'eau de la source pendant six jours et six nuits. Les Ait Ibaqalliwn quant à eux reçurent l'eau les six jours et six nuits suivants. Plus tard, les Ait Ibaqalliwn obtinrent un jour et une nuit supplémentaires pour compenser le temps que l'eau de la source met pour arriver à Ibaqalliwn. Enfin, plus récemment, Rbat d'en bas devint un village indépendant appelé Akourbi. La taqbilt de Rbat fut divisée en deux, ainsi que le territoire et ses ressources. Rbat reçu alors trois jours et trois nuits d'eau, comme Akourbi (Figure 6).

Les anciens affirment que depuis ce temps, le partage de l'eau entre les villages n'a pas été modifié. En temps de paix, le tour d'eau intervillageois est la seule règle en vigueur pour le partage de l'eau. Venant des ancêtres, cette règle ne peut pas être modifiée.

\section{Ayants droit et droits d'eau}

À travers ces récits de fondation, l'origine du partage de l'eau est décrite et justifiée. Ces récits permettent ainsi d'identifier certaines caractéristiques structurantes du droit local de l'eau, notamment à propos du groupe des Ait Tiwili, que nous traduisons par la notion de groupe d'ayants droit ${ }^{5}$.

La plupart des sources de la vallée sont situées sur des terres possédées collectivement par une taqbilt villageoise dont elles portent généralement le nom. Mais la possession des terres sur lesquelles est sise la source n'entraîne pas la possession de la source, ni celle de ses eaux par la taqbilt en question. Les Ait Bou Guemez ne considèrent pas l'eau des sources comme un bien privé, qu'il soit individuel ou collectif. Les anciens affirment que « ce qui vient de sous la terre est à Dieu ». Et, en effet, dans les récits recueillis, aucune revendication de propriété des sources n'est mise en avant, puisque ce sont des chèvres et non des ancêtres identifiés qui découvrent les sources. De même, les groupes d'ayants droit ne sont pas légitimés par l'aménagement originel du réseau d'irrigation. Pour la source de Rbat, aucun récit ne relate l'histoire de l'aménagement de l'espace irrigué : «c'est comme ça depuis que les pierres sont sur la montagne », nous dit-on. L'idée d'une appropriation privée de la source ou des canaux paraît absurde aux habitants interrogés. Cette situation contraste avec de nombreux cas étudiés au Maroc. Souvent l'existence d'une hiérarchie des droits d'usage vient justifier l'existence de parts d'eau inégales, soit en vertu de droits d'amont ou d'antériorité, soit en vertu de droits acquis lors de la construction des canaux d'irrigation (Ameur 1988).

En revanche, l'histoire de la découverte d'une source est associée dans tous les récits à la fondation des villages qui la jouxtent. Ces villages sont présentés comme ayants droit au vu de leur position spatiale et historique sur le territoire attenant originellement à la source. Ainsi, si le propriétaire de la chèvre et le berger ne sont pas identifiés individuellement, leur taqbilt d'appartenance est toujours mentionnée. Pour la source de Rbat, la chèvre appartient à un Ait Ibaqalliwn et le berger est employé par la même taqbilt. Le territoire d'Ibaqalliwn où se trouve la source est donné au berger qui fonde Rbat. Mais la source n'est pas comprise dans ce don puisque son existence est ignorée des Ait Ibaqalliwn. Par contre les eaux de la source de Rbat arrosent le territoire d'Ibaqalliwn qui fut peu à peu divisé entre les Ait Ibaqalliwn, les Ait Rbat, puis les Ait Akourbi. En tant que propriétaires de ces terres, les membres des trois villages sont d'emblée présentés comme ayants droit de la source qui les alimente. 
Le groupe d'ayants droit à la source de Rbat est donc délimité à travers le mythe, par la propriété des terres originellement alimentées par cette source. Le droit d'eau est attribué aux terres et non à leurs propriétaires ; l'eau est « mariée à la terre ». Ainsi nous l'explique un habitant de la vallée : "l'eau c'est pour la terre, pas pour les hommes. [...] Dans d'autres régions, on partage l'eau en fonction des familles. Mais dans ces régions on ne doit pas irriguer depuis longtemps, parce qu'il n'est pas normal de vendre l'eau sans la terre $»^{6}$. Dans la vallée des Ait Bou Guemez, toute parcelle située dans le fond de vallée possède un droit d'eau notifié dans les actes de vente (Figure 7). Acte de vente d'une parcelle
mentionnant le droit d'eau
qui y est attaché.

(Fig. 7)

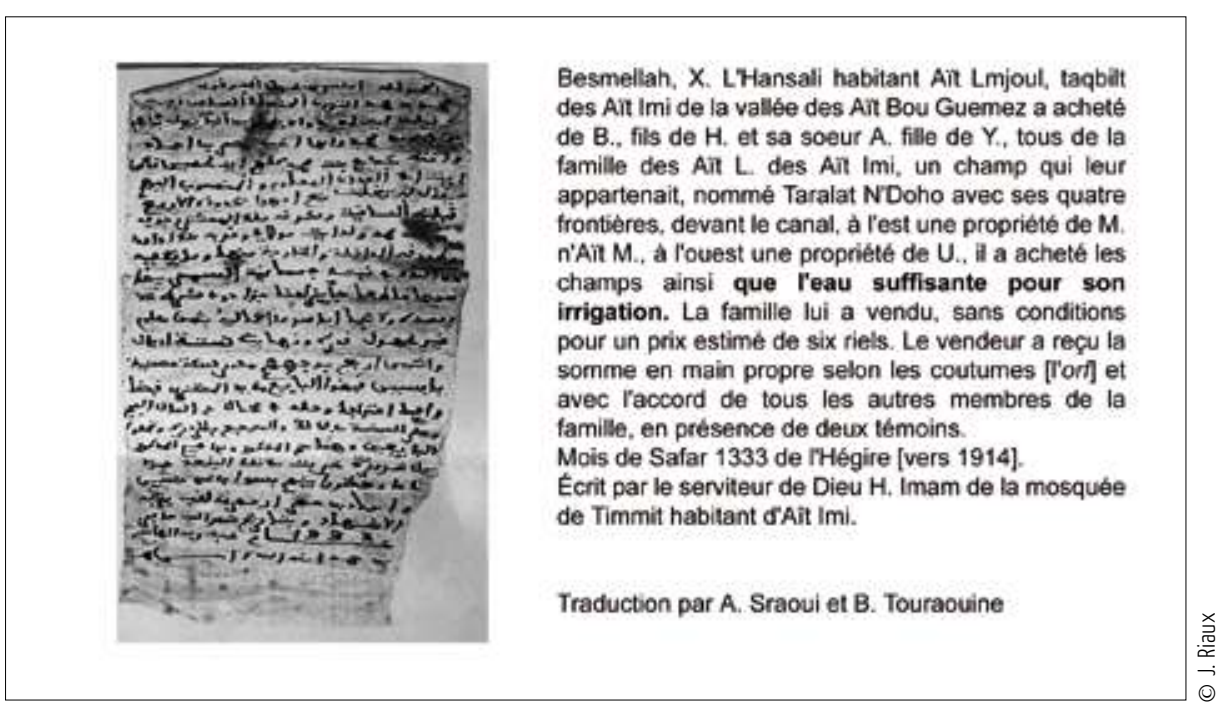

Le droit d'eau est donc défini et alloué à travers deux niveaux de droit : le droit d'eau collectif définit l'ensemble des parcelles qui ont droit à l'eau d'une source et le droit d'eau attaché à la parcelle concrétise ce droit collectif. Les termes « tiwili » et « Ait tiwili » sont utilisés aux deux niveaux de gestion : le tour d'eau entre villages du groupe sociohydraulique et le tour d'eau au sein de chaque village. Cette façon de dire le droit fait écho aux constats d'Aubriot (2002) qui distingue le droit d'eau défini comme un droit d'accès à l'eau dépendant du processus social de reconnaissance par les autres utilisateurs, et le droit d'eau comme part d'eau qui détermine implicitement une personne la possédant comme ayant droit.

Par ailleurs, le droit est présenté comme étant strictement égalitaire. D’une part, chaque parcelle située sur le territoire d'un village ayant droit possède un droit à l'eau. Ce droit n'est pas exprimé en débit ou en temps mais en quantité d'eau que la parcelle reçoit à son tour. Cette quantité est estimée à une lame d'eau de la hauteur d'une cheville. Chaque parcelle de l'aire sociohydraulique a donc droit à une part d'eau théoriquement proportionnelle à sa surface. D'autre part, chaque groupe villageois à un droit d'eau équivalent en temps, ce qui a d'ailleurs été ajusté au cours du temps avec l'octroi d'un jour supplémentaire à l'aval pour tenir compte du temps de circulation des eaux. Ce partage égalitaire est justifié dans les récits. Il est en effet admis que la taqbilt des Ait Ibaqalliwn a été la première installée sur le territoire de la source de Rbat. Les Aitt Ibaqalliwn étaient donc chronologiquement les premiers bénéficiaires de la source plus tard usurpée par 
les Ait Rbat situés en amont du territoire alimenté par la source. On peut donc penser que le mythe justifie l'impossibilité morale d'appliquer un droit d'amont, puisque c'est le village d'aval qui a offert la source à ceux de l'amont arrivés ensuite. Il y a à la fois annulation des droits d'amont et des droits d'antériorité.

Les récits concernant la pacification de la vallée offrent une version détaillée du processus qui a abouti à la négation du droit d'amont par la mise en place du « tour d'eau ».

\section{De la guerre au contrat}

La pacification a été vécue par les anciens de la vallée ou par leurs parents. Les récits concernant cette période se situent entre épopée historique et souvenirs anecdotiques de la pacification de la vallée par les armées du Protectorat, conduites dans le Haut Atlas par le Glaoui de Marrakech. Cet épisode de la chronique tribale est essentiel puisqu'il marque le passage du temps de «siba (la guerre) à celui de « thena » (la paix). C'est aussi le passage d'une organisation tribale fondée sur l'autorité des Zaouias et des chefs tribaux, à une soumission au Makhzen imposée localement par de puissants cailds nommés par les officiers du Protectorat. Le récit de cette transition historique est divisé en trois périodes : le temps de «siba » précède l'entrée des armées du Makhzen dans la vallée ; "l'arrivée du Glaoui » désigne la période de pacification armée ; enfin, la « colonisation » correspond à la période de présence des français.

Le temps de «siba » est marqué par d'incessants conflits entre les villages. Les anciens racontent qu'à cette époque la zaouila Ahansal avait une influence à la fois politique et religieuse sur les Ait Bou Guemez, et particulièrement sur les Ait Hakim soumis à son autorité. Le caïd Ahansal était chargé de régler les conflits concernant l'amont de la vallée. Les conflits relatés sont multiples, tant entre villages qu'entre groupes de villages rassemblés en leffs. Selon nos interlocuteurs, Ibaqalliwn était constamment en conflit avec les villages voisins. En raison de ces conflits, les Ait Ibaqalliwn étaient alliés aux Ait Ouanougdal du vallon des Ait Hakim, ces derniers étant eux aussi en conflit avec leurs voisins. D’autre part, le leff des Ait Hakim était opposé à celui des Ait Mihiya de l’aval des Ait Bou Guemez (Figure 8).

\section{Une vallée divisée}

Les récits de la pacification cristallisent les alliances et oppositions politiques de l'époque.

(Fig. 8)

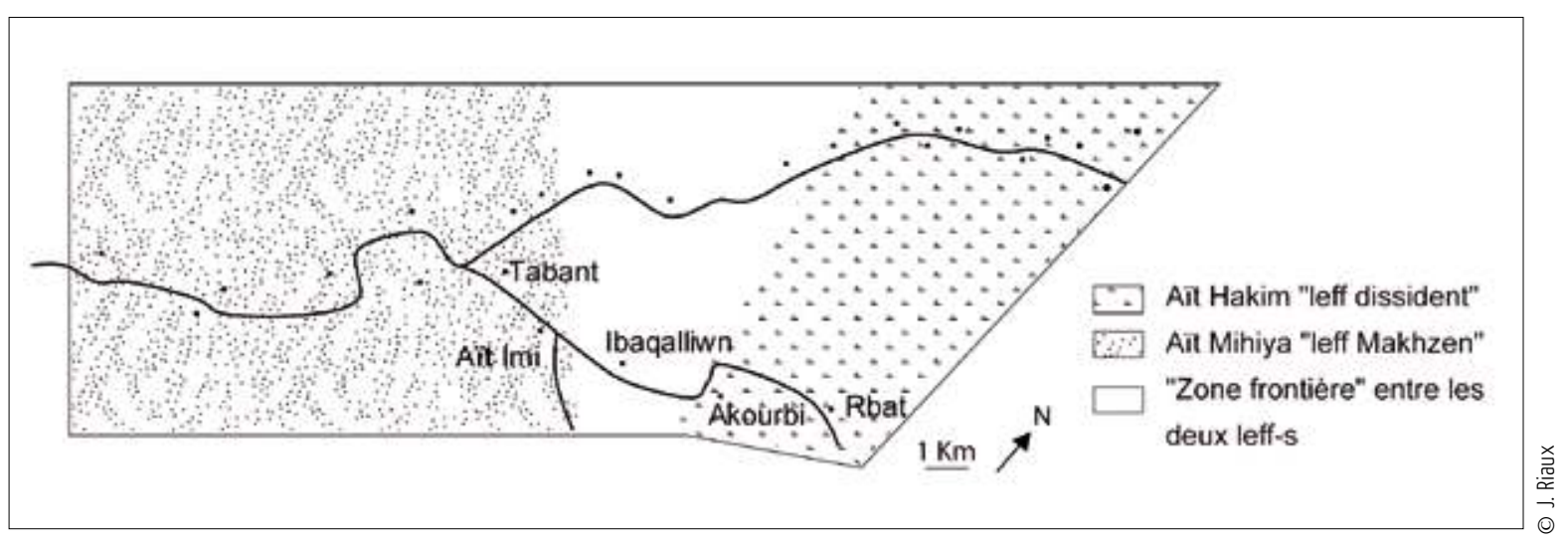


À travers les récits détaillant conflits et inimitiés entre ces groupes, deux aspects de l'histoire se font jour. D'une part les alliances entre villages étaient fluctuantes, particulièrement dans la zone centrale de la vallée. D'autre part, une opposition claire existe entre les Ait Hakim et les Ait Mihiya. Les villages géographiquement et politiquement situés entre ces deux groupes, s'alliaient donc à leurs voisins en fonction des intérêts du moment.

Les récits concernant la période de pacification ne prennent pas en compte le laps de temps écoulé entre la soumission des populations de l'aval en 1916 et celle de l'amont en 1923 (Lecestre-Rollier 1992). Cette période est illustrée par un unique épisode guerrier : l'arrivée du Glaoui dans la vallée. Plusieurs versions de cet événement existent. Selon les anciens d'Ibaqalliwn, le Glaoui trouva leur village vide, car ils s'étaient réfugiés chez leurs alliés pour préparer la bataille. Les Ait Ibaqalliwn auraient fait la guerre aux Ait Mihiya aux côtés des Ait Hakim. Mais les anciens d'Akourbi et de Rbat racontent que le Glaoui a brûlé le village d'Ibaqalliwn et mis les Ait Ibaqalliwn sous ses ordres. Ensuite, les Ait Ibaqalliwn auraient brûlé le village d'Akourbi sous les ordres du Glaoui. Le lendemain, et tous nos interlocuteurs le relatent, a eu lieu une grande bataille sur la montagne Adazen. Les Ait Hakim et leurs alliés ont gagné cette bataille. Lorsque le Glaoui s'est enfui, les Ait Hakim ont à nouveau brûlé le village d'Ibaqalliwn.

Pour les gens du vallon de Rbat, l'aspect actuellement signifiant de cette période est l'opposition entre villages et ses conséquences en termes de partage de l'eau. Comme nous l'explique un ancien d'Akourbi :

« je ne connais pas l'origine de cette guerre, mais ce qui est important c'est qu'il y a eu une guerre entre les Ait Rbat et les Ait Ibaqalliwn, et qu'elle a duré sept ans. Alors c'est pour ça qu'il y a toujours des disputes et que les Ait Rbat coupent l'eau aux Ait Ibaqalliwn».

On assiste aujourd'hui à une cristallisation des relations d'oppositions qui existaient au temps de l'arrivée du Glaoui. Pour l'ensemble des Ait Bou Guemez, deux grands groupes existent : les Ait Mihiya et les Ait Hakim, vestiges du «leff makhzen » et du « leff dissident » (Figure 9). De nombreux conflits concernant les ressources collectives recouvrent actuellement cette opposition : les Ait Rbat contre les Ait Ibaqalliwn, les Ait Ouanougdal contre les Ait Hakim. Ainsi l'opposition politique épouse-t-elle une dichotomie amont/aval en termes de partage de l'eau.

Dans les récits recueillis, l'arrivée des Français dans la vallée marque un changement. Tout d'abord, ces derniers auraient mis fin à l'influence religieuse et politique des zaouias. Ce sont désormais les officiers du Protectorat qui arbitrent les conflits liés à l'exploitation des ressources collectives. En cas de conflit les territoires villageois et tribaux sont délimités, des tours d'eau pour l'irrigation sont instaurés. Les anciens de Rbat, Akourbi et Ibaqalliwn s'accordent sur le fait que c'est Rbat qui a gagné la guerre et qu'à l'issue de cette période les Aït Ibaqalliwn n'avaient plus l'eau. C'est alors que les Français auraient suggéré aux Ait Ibaqalliwn d'égorger un bœuf chez les Ait Rbat pour leur demander un droit d'eau. Ce fut fait. Un pacte fut scellé par le sacrifice de l'animal et la règle du tour d'eau fut mise en œuvre. L'histoire dit aussi que les Ait Rbat ayant acheté entre temps des terres aux Ait Ibaqalliwn avaient tout intérêt à laisser passer l'eau vers ce terroir. Entre Rbat et Ibaqalliwn le tour d'eau est en vigueur depuis cette époque là, même si en pratique le non-respect individuel du tour d'eau fait l'objet de conflits récurrents. 


\section{Un partage contractuel, égalitaire et légitime}

Les mythes de fondation et les récits concernant la pacification sont racontés de manière différente. Le mythe de fondation des villages est positionné dans un temps anhistorique, tandis que la pacification a été vécue par certains de nos interlocuteurs. Elle est datée et présentée comme telle. Cependant, les deux récits coïncident sur le fait que le pacte de partage de l'eau a été conclu à la fin d'une guerre de sept ans. Ainsi ces récits placent-ils l'origine de la règle de partage de l'eau à la fois dans le temps des origines et dans le temps historique. La règle apparaît alors doublement légitimée puisqu'elle repose sur un pacte conclu par les ancêtres et validé par une autorité extérieure à la vallée.

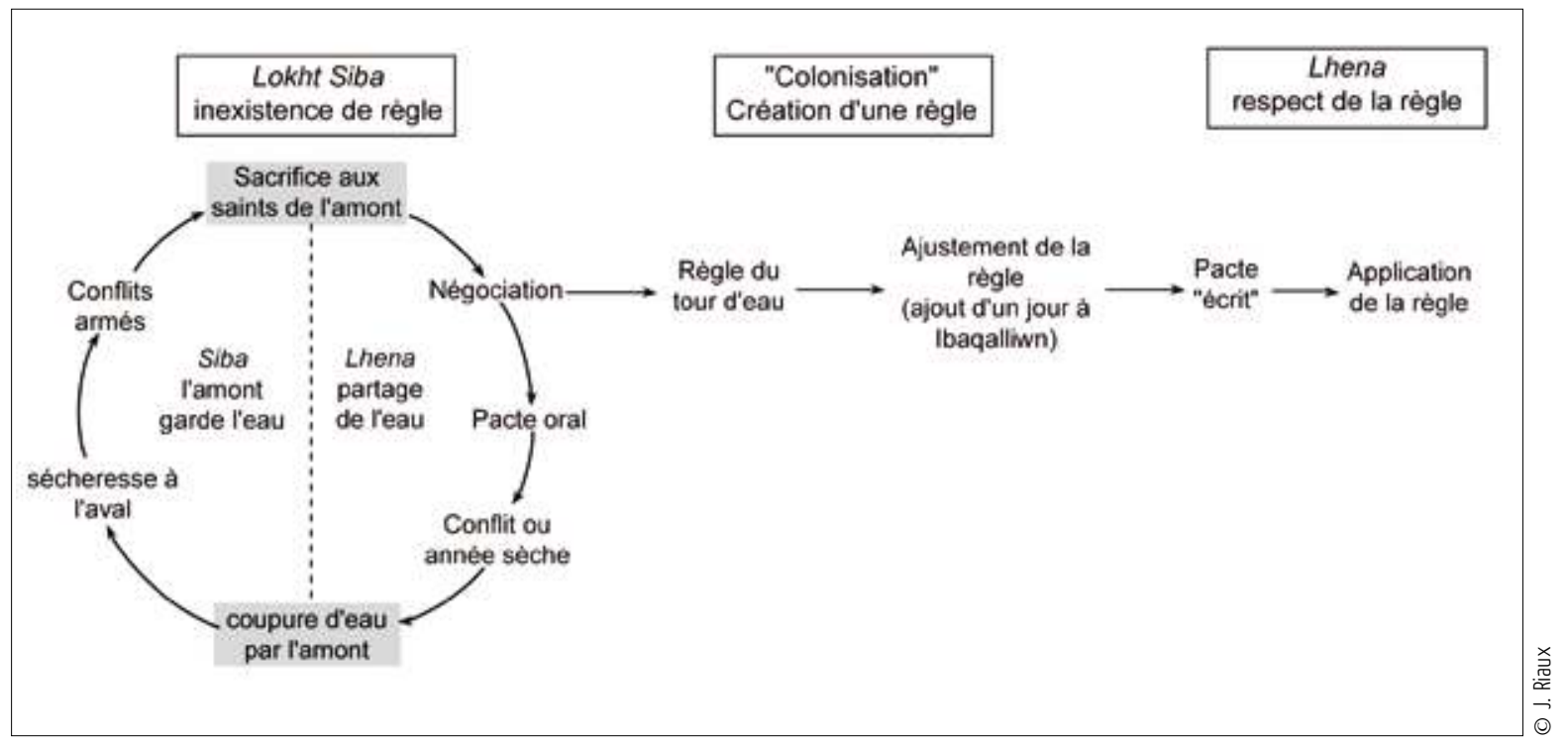

Genèse mythique de la règle de partage de l'eau entre Rbat et lbaqalliwn

La signification des termes « siba » et « Ihena » est double. L'une apparait dans le temps cyclique : au temps de siba, des périodes de guerre et de paix alternaient. L'autre comporte un aspect définitif : au temps de la colonisation, on passe de siba à /hena. L'accord sur le partage de l'eau représente le passage d'un ordre ancien à un ordre moderne. On passe de la même manière du récit mythique au récit historique.

(Fig. 9)

L'existence du tour d'eau garantit un accès à l'eau à tous les villages ayants droit, quelles que soient les relations intervillageoises en cours. En cela l'accord fixant la règle de partage représente un aboutissement : l'instauration d'une stabilité durable. Cet aspect, récurrent dans le récit des origines, est exprimé à travers le passage de «siba » à « thena »: de la guerre à la paix, de l'anarchie à la règle. La création d'une règle de partage de l'eau oppose donc un temps linéaire au temps cyclique qui l'a précédé (Figure 9).

Le pacte instaurant le tour d'eau a pour fonction de réguler l'accès à l'eau afin que la pratique collective de l'irrigation soit possible. Dans ce cas précis, pour être respecté, un contrat doit être acceptable par toutes les parties en présence. Dans ce but, il doit être équitable et c'est bien ce que revendiquent les «signataires» du pacte. La définition originelle des parts d'eau est présentée comme résultant d'un consensus: 
chaque village à un droit d'eau équivalent. On aboutit donc à une règle théoriquement égalitaire. C'est bien ainsi que les ayants droit de la source de Rbat présentent le tour d'eau, tout en affirmant son caractère ancestral. Ainsi, les règles intervillageoises de partage de l'eau n'ont jamais été remises en question lors de nos enquêtes : " nos ancêtres ont fait ça et on continue ce qu'ils ont commencé ». D'ailleurs, cette alliance contractuelle a longtemps été réaffirmée à travers la vie rituelle. Chaque année, les trois taqbilts achetaient ensemble une vache noire qui était sacrifiée à la source de Rbat.

\section{$\&$}

Dans la vallée des Ait Bou Guemez, l'organisation du partage de l'eau est énoncée, justifiée et légitimée à travers la conjonction de récits relevant du mythe pour l'un et de la chronique locale pour l'autre. Ces récits recouvrent bien plus que le seul partage de l'eau. Il s'agit avant tout de relater l'histoire d'un groupe et l'émergence du pacte qui fonde des relations contractuelles. L'eau rassemble alors, au-delà des divisions qu'elle suscite.

Les récits véhiculent les règles qui encadrent l'usage collectif de l'eau et en particulier pour l'irrigation : limites du groupe et de l'espace, modalités d'attribution de parts d'eau à ces ayants droit collectifs et individuels. Outre l'exposé de règles, ces récits en expliquent la genèse et la raison d'être ainsi que les principes sur lesquels repose leur légitimité : contrat, équité, ancestralité. Il ne s'agit pas seulement d'une liste de prescriptions comme c'est le cas dans un règlement d'arrosage, mais bien d'un corpus de règles qui fonde le droit local de l'eau. Si l'on ne prend pas ces récits en compte dans l'écriture des règles de gestion de l'eau, on oblitère la cohérence d'ensemble de l'édifice juridique ainsi énoncé.

Cela nous renvoie aux problèmes posés par la formalisation des règles de gestion de l'eau. Il n'y a pas ici un discours clairement dédié à l'explicitation de ces règles sur lequel pourraient s'appuyer les agents en charge de la formalisation. L'énonciation du droit de l'eau ne repose pas sur un format de discours spécifique comme c'est le cas lorsque ce droit est écrit. La reconstitution d'un corpus de droits relèverait ici de l'interprétation des récits et de la classification des éléments qu'ils contiennent, activités que les Ait Bou Guemez n'effectuent pas pour expliquer le partage de l'eau. Dès lors, formaliser les règles existantes comme c'était prévu dans la vallée des Ait Bou Guemez passe par une prise en compte de ces récits, puis par une traduction en règles inscriptibles dans des registres écrits. Mais, pour l'instant ces récits demeurent dans une sphère extérieure aux projets de modernisation des réseaux hydrauliques ; ils sont relégués dans le domaine du folklore à la fois par les intervenants extérieurs et par les irrigants qui, interrogés sur les règles de gestion, ne racontent pas ces récits.

Un travail sur les modalités de production et de transformation de ces récits permettrait d'interroger les rapports de force qui sous-tendent l'appropriation et la transmission de ce type de récit. De même, la réinscription de ces récits dans leur contexte d'énonciation, notamment en regard des pratiques contemporaines de partage de l'eau et des enjeux relatifs à l'intervention publique sur les droits, serait un moyen privilégié de restituer aux récits leur efficacité pratique (Bensa, 2006). 


\section{NOTES}

Photo d'ouverture : La source de Rbat. Il y a en réalité deux sources à Rbat : l'une pérenne, l'autre saisonnière. Si elle coule assez tôt au printemps, cette dernière assure une année d'abondance.

1. Ces récits ont été recueillis au cours d'une vingtaine d'entretiens menés entre 2001 et 2003 auprès des ancien(ne)s des villages étudiés. Des habitants ont désigné les personnes les plus à même de transmettre l'histoire et ont assisté aux entretiens, ajoutant ou contestant certains détails. Les récits ont été recueillis dans la langue locale avec l'aide de A. Sraoui, O. Bouharazen et H. Afsahi, enregistrés, puis traduits et comparés. Les habitants des villages étudiés ont validé la version que je proposais de leurs récits lors d'un exposé public.

2. Ces récits n'appartiennent pas au domaine de l'histoire sacrée mettant en jeu les êtres surnaturels et les fondements de l'existence. Ils ne sont donc pas des mythes de fondation au sens où l'entend Eliade (1963). Par contre, ils jouent un rôle analogue au mythe défini par cet auteur : « le mythe raconte comment, grâce aux exploits des êtres surnaturels, une réalité est venue à l'existence, que ce soit la réalité totale, le Cosmos, ou seulement un fragment : une île, une espèce végétale, un comportement humain, une institution ».

3. La notion de taqbilt désigne toute unité socio-spatiale d'ordre politique (Lecestre-Rollier 1992). À l'échelon villageois, le plus prégnant aujourd'hui, la taqbilt est matérialisée par l'ensemble des chefs de foyer, qui se réunit chaque semaine pour prendre des décisions concernant la communauté, dont la gestion de l'eau. Nous traduisons ici le terme ighs par lignage, à l'instar d'Amahan (1998). Mais ce terme désigne aussi des groupements fondés sur l'appartenance territoriale et non sur des liens de parenté. Une des spécificités de la gestion de l'eau chez les Ait Bou Guemez est justement la faible mise en avant des relations de parenté au profit de relations territoriales et politiques. Les Aït Bou Guemez se distinguent ainsi des communautés pour lesquelles partage de l'eau et relations de parenté sont étroitement associés (Berque 1955, Hall 2008).

4. Les Ait Tiwili sont les personnes inclues dans le « tiwili nou'aman », littéralement : tour de rôle de l'eau.

5. Il existe pourtant dans la vallée des villages où l'eau n'est pas mariée à la terre, où les droits d'eau sont lignagers. On peut faire l'hypothèse que le mode de gestion observé résulte de transformations assez récentes que l'histoire mythique viendrait justifier. 


\section{RÉEERENCES}

Amahan, A. 1998 Mutations sociales dans le Haut Atlas, les Ghoujdama. Paris : Éditions de la Maison de sciences de l'homme, Rabat: La Porte.

Ameur, M. 1988 Le Statut juridique de l'eau et l'irrigation au Maroc. Rabat : université Mohamed V (thèse de doctorat).

Assier-Andrieu, L. 1980 Anthropologie de la coutume dans les Pyrénées catalanes françaises. Paris : Éditions de l'Ehess (thèse de doctorat).

Aubriot, O. 2002 Société et concept de droits d'eau en irrigation : appropriation ou partage de l'eau ? In O. Aubriot \& G. Jolly (dir.) Histoire d’une eau partagée. Provence, Alpes, Pyrénées. Aix en Provence : université de Provence, 35-60.

Bédoucha, G. (dir.) 2000 Prégnance du droit coutumier, Études Rurales 155-156.

Bensa, A. 2006 La Fin de l'exotisme. Essai d'anthropologie critique. Toulouse : Anarchis.

Berque, J. 1978 [1955] Structures sociales du Haut Atlas. Paris : PUF.

Boelens, R., Bustamante, R. \& Vos, (de) H. 2007 Legal pluralism and the politics of inclusion : recognition and contestation of local water rights in the Andes. In B. Van Koppen \& M. Giordano, Communitybased water law and water resource management reform in developing countries : 96-113.

Bruns, B.-R. \& Meinzen-Dick, R. 2000 Negotiating water rights. New Delhi : Vistaar Productions.

Éliade, M. 1963 Aspects du Mythe. Paris : Gallimard.

Garrigues-Cresswell, M. \& Lecestre-Rollier, B. 2001 Gérer les aléas. Les sociétés du Haut Atlas marocain, Techniques et Culture 38 : 69-95.

Hall, I. 2008 Un Canal comme support mnémotechnique pour la généalogie ?, Techniques et Culture 50 : 256-281.

Lecestre-Rollier, B. 1992 Anthropologie d'un espace montagnard les Ayt Bou-guemez du Haut Atlas marocain. Paris : Presses universitaires de Paris Sorbonne (thèse de Doctorat).

- 1999 La Loi, le pouvoir et les acteurs : jeux et enjeux au Maroc. In J.-L Jamard, A. Montigny \& F.-R. Picon Dans le sillage des techniques, hommage à Robert Cresswell. Paris : L'Harmattan, 295-331.

Ostrom, E. 1992 Crafting institutions for self-governing irrigation systems. San Francisco : ICS Press.

Riaux, J. 2006 Règles de l'État - règles de la communauté : une gouvernance locale de l'eau : vallée des Ait Bou Guemez (Haut Atlas - Maroc), Plaine de Vinça (Pyrénées - France). Paris : Éditions de l'Ehess (thèse de doctorat).

— 2011 Faut-il formaliser les règles de gestion de l'eau ? Une expérience dans le Haut Atlas, Cahiers Agricultures $20: 67-72$.

Ruf, T. 1995 Cinq siècles de conflits sur l'eau dans les Andes équatoriennes : fondation de réseaux et partage de l'eau à Urcuqui. In R. Waast (dir.) Les Sciences hors d'occident au $x x^{e}$ siècle, Nature et environnement, vol. 3 : 195-221.

Tozy, M. \& Mahdi, M. 1990 Aspects du droit communautaire dans l'Atlas marocain, Droit et Société 15 : 219-227. 


\section{RÉSUMÉ}

Dire le droit local de l'eau. Le droit local de l'eau a fait l'objet de nombreuses recherches. Au Maghreb, une place centrale est accordée aux questions relatives à la pluralité des sources du droit, ainsi qu'aux écarts entre règles énoncées et pratiques effectives. Aujourd'hui, les recherches s'orientent de plus en plus sur les problématiques liées à l'intervention publique en matière de changements institutionnels et de révision des droits locaux. Cet article s'inscrit à la croisée de ces deux orientations de recherche. L'analyse des modalités d'énonciation des règles de partage de l'eau, dans une société de tradition orale, a pour objectif de souligner la complexité des systèmes de droits locaux et leur imbrication dans l'histoire politique locale.

Dans le Haut Atlas central, les Ait Bou Guemez organisent de longue date la gestion et le partage de l'eau en fonction de règles produites localement. L'origine de ces règles, le tour d'eau notamment, est racontée à travers deux principaux récits. En premier lieu, les mythes de fondation des villages, associés à la découverte d'une source racontent l'origine du groupe d'ayants droit et l'histoire du partage des eaux de cette source. Ensuite, le récit de la période de pacification de la vallée par les forces armées du Protectorat relate l'origine du contrat qui donna lieu au tour d'eau en vigueur jusqu'à aujourd'hui. Temps mythique et temps historique sont ainsi sollicités pour expliquer et légitimer les règles contemporaines de partage de l'eau.

\section{ABSTRACT}

Narrating water rules and rights. Many studies have been conducted on the issue of local water rules and rights. In the North African studies, the two main subjects related to this issue are the legal pluralism and the differences between rules and practices. But on present days, studies are focusing on issues related to the public interventions on local irrigation systems, as institutional changes and revision of local water rights. Our contribution establishes links between these two research fields through the analysis of the way an irrigation community enounces the local water rights. This paper underlines the complexity of the systems of rights and explains their interweaving in the local history.

In the Central High Atlas (Morocco), the Ait Bou Guemez people have a long background of collective water management. There, collective organization is based on rules locally and historically crafted. The origins of these rules, the water turn in particular, are told through two main "stories". The first one is about the founding myths of villages around the discovery of a spring. Through this type of "story", the Ait Bou Guemez people explain the origins of the stakeholders group and the "story" of water-sharing. The second kind of story describes the period of Pacification of the valley by the French Protectorate army and the origins of the water turn, resulting of an agreement obtained in a situation of conflicts. Thus, mythical time and historical time are used to make links between contemporary practices and the history of the valley, and to legitimate water-sharing rules in use.

\section{MOTS CLÉS}

Haut Atlas, Ait Bou Guemez, droit local, irrigation, tour d'eau, histoire orale

\section{KEYWORDS}

High Atlas, Ait Bou Guemez, customs, irrigation, water turns, oral history 